\title{
The Teaching Laboratory Data Management (TLDM) System
}

\author{
Derek Yau Chung Choy, Jim Sibley, and Dhaneshwarie Kannangara \\ The University of British Columbia \\ dhanesh.kannangara@ubc.ca
}

\begin{abstract}
In the teaching laboratory, students generate large amounts of data and often struggle with the subsequent calculations for results. Grading the substantial amounts of results and calculations from a large class is very taxing for teachers, who are left with less time to interact with students. The TLDM system aims to resolve the primary challenge associated with laboratory experimental calculations: while the numerical operations for a given experiment are expected to be the same, the correct numerical results vary based on the unique raw data collected by each student. The system provides scalable instructional scaffolding to guide students through their own calculations. The system also works to generate custom marking keys unique to each student's raw data to assist teachers in grading the numerical component of reports, leaving them with more time to provide feedback in other areas.
\end{abstract}

Keywords: TLDM, Teaching Laboratory, Data Management, Microsoft Excel ${ }^{\mathrm{TM}}$, Flexible Learning, Scaffolding, Fading

\section{INTRODUCTION}

Teaching laboratories play an important role in engineering and science education, most often serving a complementary role to classroom instruction [1-3]. The typical teaching lab consists of two major components: 1) the laboratory portion that provides an environment for students to gain experimental skills in situations that provide real examples of the scientific concepts they are learning, and 2) the report portion that requires students to analyze data gathered in the laboratory and discuss relationships between theoretical models and real behaviour. Both components involve the handling of experimental data, which is collected in the laboratory and used to calculate results that are the basis for discussion in the report. Usually, this data is numerical and is unique to each student or group performing each experiment in the laboratory course. As a result, a large amount of data is routinely generated over the years. This paper describes a method for organizing, manipulating and storing this data to facilitate student learning, reduce teacher workload and assist in course development.

\section{CHALLENGES}

There are challenges associated with managing the large amounts of raw data and the complex calculations that must be completed for many lab reports. The uniqueness of each data set creates a large challenge for teachers when they review and grade reports. Detailed grading by the teacher requires the calculation of unique correct results from each unique data set, which can be very taxing for teachers. As the grading workload intensifies, it is increasingly difficult to detect issues such as data "fudging" or plagiarism within the sea of numbers and calculations. Finally, as calculations become lengthier and more complicated, students themselves also experience increasing difficulties navigating the plethora of numbers and calculations. This forms a vicious cycle: as students require more support from teachers, the teachers themselves have less time to provide the necessary timely feedback.

In spite of all the challenges in dealing with large amounts of lab data, there are also new opportunities that can benefit both teachers and students. When properly managed, this vast amount of data contains valuable information that can be used to track equipment performance, student performance, teacher effort, and can also help with continual course improvement. The key is collect as much data from the classroom as possible to build a large data set, much like big data is being collected at a global scale and further processed at a later time to answer questions and solve problems that may come up at a later time. A major challenge is the efficient collection, organization, and storage of collected data. With the aid of powerful computers and elaborate software, big data is already playing a key role in industry, but these strategies can be too resource intensive and too difficult to customize for easy application to engineering education. Data management strategies that are leaner and more flexible are needed for the much smaller "big data" that is collected in the classroom.

\section{SOLUTIONS}

In this article, we describe the Teaching Laboratory Data Management (TLDM) system that manages these challenges and provides benefits for both students and teachers. The system is based on Microsoft Excel ${ }^{\mathrm{TM}}$, a powerful spreadsheet application that is widely used in engineering education [4-6]. The TLDM system is currently used in $2^{\text {nd }}$ and $3^{\text {rd }}$ year level teaching laboratories at the Department of Chemical and Biological Engineering (CHBE) at The University of British Columbia. How the system works, feedback from students and teaching assistants on the pilot introduction of the system in a junior 
laboratory course, and the subsequent introduction of the system customized for a more senior level laboratory course will be discussed.

\subsection{Teaching Laboratory Data Management (TLDM) system - Aims}

The TLDM system aims to:

- Help students with the collection of raw data from experiments and accurately perform the subsequent numerical calculations to complete each lab report.

- Help teachers grade the numerical results in lab reports with more accuracy and with reduced effort.

- Facilitate the compilation and use of laboratory big data for long-term improvements of the teaching laboratory.

\subsection{Teaching Laboratory Data Management (TLDM) system - File structure}

The TLDM system is based on series of Microsoft Excel $^{\mathrm{TM}}$ files. We chose Excel $^{\mathrm{TM}}$ since it is easily accessible for both teachers and students. The TLDM system provides students with guided pathways, with instructional scaffolds and feedback, as they grapple with long complex laboratory analysis and calculations. It also provides grading assistance for teachers by automatically generating marking keys unique to each student's unique raw data. The system consists of 5 Excel $^{\mathrm{TM}}$ files:

- Raw Data (D) file - contains pre-formatted tables for students to record raw data generated in the laboratory.

- Calculations Template file: contains pre-formatted tables containing a set of sample raw data and instructions that guide students on how to enter the appropriate formulae to calculate final results, generate plots/figures, and develop their calculation strategy.

- Answers to Sample Raw Data file: contains sample raw data and the correct values of results derived from the sample raw data to allow students to check their calculation strategy.

- Final Answers (A) file: contains pre-formatted tables for students to enter final calculated results derived from their unique raw data.

- Master Grading (C) file: a version of the Calculations Template file that contains the correct formulae required to generate final results for any set of input raw data (only available to teachers).

\subsection{Teaching Laboratory Data Management (TLDM) system - Workflows}

The Teaching Laboratory Data Management (TLDM) workflow consists of seven steps, as illustrated in Figure 1. The first five steps are intended for students while the final two steps are completed by teachers:
1. Students review the $\mathbf{D}$ file before the experiment to see what raw data is expected to be collected.

2. Students perform the experiment and enter their raw data into the D file, which is submitted at the end of each lab class so that values cannot be "fudged" later.

3. Students begin building formulae in the Calculations Template file using the provided sample raw data and corresponding results. They check their calculated results against those found in the Answers to Sample Raw Data file as a means of receiving instant feedback to validate the accuracy of their calculation strategy. Students can iteratively develop their calculation strategy at their own pace until the formulae they use yield the correct results.

4. After students are satisfied with calculations strategy they developed in Step 3, they enter their own raw data into the completed Calculations Template file to obtain the final results for their own experiment.

5. Students enter their final results into the $\mathbf{A}$ file. This file is preformatted to clearly show students what results are required for the experiment, and to allow instructors to easily locate the values while grading. Students submit the A file along with their lab report.

6. Instructors use the originally submitted $\mathbf{D}$ file and the C file to auto-generate the unique answer key and grading sheet from the raw data from each student.

7. The unique answer key and auto-generated grading sheet are used to mark the report. The key highlights deviations from A file values to assist grading.

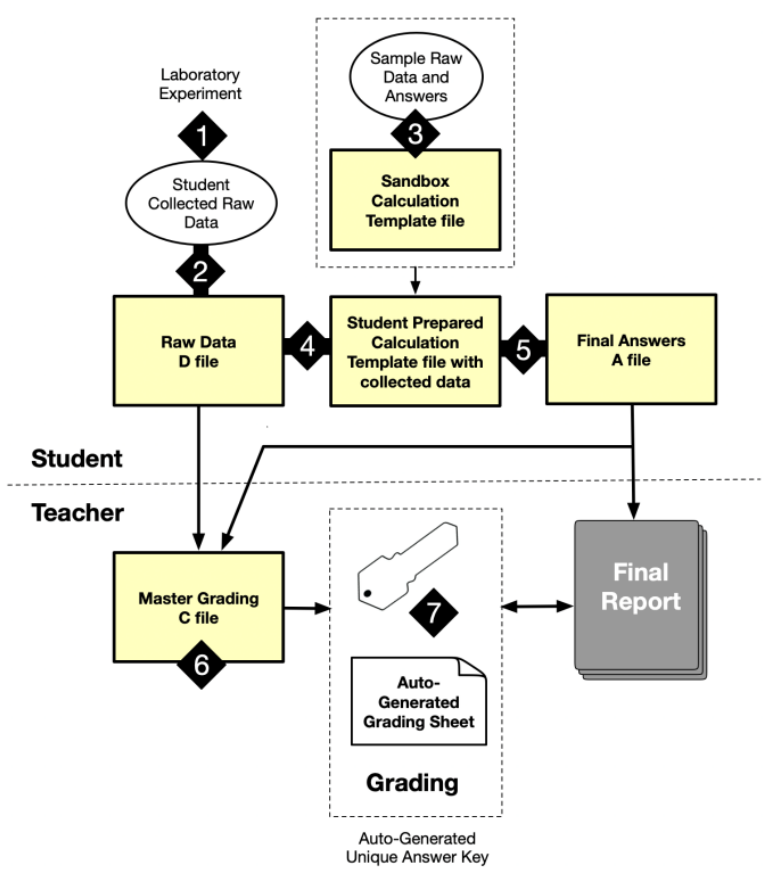

Figure 1: Teaching Laboratory Data Management (TLDM) system flow diagram 
The contents of the files described above are unique to each laboratory experiment, and are designed to accommodate any variations in student data generated in the classroom as long as they follow the same set of experiment instructions outlined in the laboratory manual.

\section{BENEFITS TO STUDENT LEARNING}

The best learning occurs when students feel engaged [710] and tasks are at an appropriate level of difficulty. Bjork [11] has coined the phrase "desirable difficulty" to describe how task difficulty can be structured to challenge but not overwhelm students to maximize their learning. When dealing with difficult tasks such as complex calculations, many students lose motivation as they struggle with critical milestones or get lost halfway through the arduous path. Controlling the level of support and feedback to keep the difficulties "desirable" and providing guided pathways to success was our primary driver for the development of the TLDM system.

\subsection{Guide Pathways}

We believe that students, especially in their junior years, should be focused on understanding scientific concepts, and not with formatting Excel ${ }^{\mathrm{TM}}$ spreadsheets or mindlessly grinding through calculations. By providing guided pathways with pre-built and pre-labeled data tables and important major intermediate values required to build towards correct final results, we can keep students on track to complete complex calculation pathways. Detailed stepby-step instructions, cross-referenced with specific cells in the data tables, hints, tips, and information, are also included in the Calculations Template file to provide additional scaffolding. All of these features work together to help reduce student cognitive load when they are tasked with complex calculations, so that they can focus more on understanding the scientific and mathematical concepts associated with the experiments.

\subsection{Managing difficulty and providing timely, focused feedback}

The difficulty of a given assignment or task can have a large effect on student engagement and motivation [12]. Reducing complexity can sometimes help students stay engaged, but reducing the difficulty of the required calculations in a laboratory experiment is sometimes not possible, as some components are essential and cannot be easily adjusted or omitted. Setting the appropriate level of difficulty has always been a challenge for educators, and is especially tough for laboratory courses with large classes where students can have a wide range of prior experience and competence levels. The TLDM system enables learning to occur at a pace that is right for each student, thereby eliminating the need for the entire class to learn at the same rate.

The Calculations Template file containing a set of sample raw data which, together with the Answers to Sample Raw Data file, forms a virtual classroom for students to learn at their own pace. In the traditional laboratory course, students have to perform a series of calculations to obtain correct final results to include in their lab reports; the entire process is very much like a one-way obstacle course where one must successfully clear each and every hurdle along the way in order to successfully complete the lab. As many calculations require correct input values from previous calculation steps, students who fail to successfully complete an early calculation step find themselves unable to reach the end even if they had no issues with some subsequent steps; to make matters worse, the successful completion of their lab reports also requires the complete set of correct results. They have no way of finding out whether they had made a mistake in their calculations, and where the error occurred, until after they receive their graded reports, which is usually quite some time after they complete their experiments. The purpose of providing the Answers to Sample Raw Data file is to provide instant feedback for students while they attempt to come up with the correct calculations process. The Calculations Template file is a virtual sandbox for students to try their calculation strategies in a safe environment using the sample raw data. When their calculated results differ from those provided in the Answers to Sample Raw Data file, they are free to try again and again until they get it right, anytime and anywhere they have access to a computer with Microsoft Excel $^{\mathrm{TM}}$ installed. When they are satisfied with their calculations strategy, they can use their own raw data to generate their lab results, which they can then confidently use to write their final reports.

\subsection{Scaffold fading}

To accommodate students with various backgrounds and in different years of the program, the scaffolding [1315] offered by the TLDM system is adaptable, progressive and, more importantly, optional [12]. All of the tip prompts in the Calculations Template file can be sequentially removed as part of a fading strategy for more advanced students. For example, the amount of instructions included in the file can be reduced or eliminated, leaving only the pre-built data tables as scaffolds; eventually these tables can also be removed. As students progress to more senior years, they need to be able to complete these calculations independently with little external help. Students in the final year of using the TLDM system can expect to be left with only the sample raw data and the answers to sample raw data, which they can still use for self-directed learning. One possible fading scheme for the TLDM system is based on the progressive removal of scaffolding, as shown in Table 1 below: 
Table 1: Possible Scaffold Fading Scheme

\begin{tabular}{|l|c|c|c|}
\hline Undergraduate Year & 2 & 3 & 4 \\
\hline Sample Raw Data & $\checkmark$ & $\checkmark$ & $\checkmark$ \\
\hline Answers for Sample Raw Data & $\checkmark$ & $\checkmark$ & $\checkmark$ \\
\hline Labeled and Formatted Data Tables & $\checkmark$ & $\checkmark$ & \\
\hline Step-by-Step Annotated Instructions & $\checkmark$ & & \\
\hline Partially-Prepared Figures & $\checkmark$ & & \\
\hline Discussion of Sample Results & & & $\checkmark$ \\
\hline
\end{tabular}

Most importantly, all of the scaffolds provided by the TLDM system, including the use of the sample raw data, are optional such that they will not interfere with the learning of more expert learners, which is always a concern when using scaffolds and performance supports [16]. Very often, elaborate scaffolds designed to help weaker students can be a hindrance to stronger students. Students are never asked to submit their Calculations Template file, and are only evaluated based on the quality of their lab report, meaning that students are always free to use their own calculation methods as long as they obtain the correct results.

\subsection{Unified lexicon for academic discourse}

The TLDM system can contribute significantly towards improving the clarity and effectiveness of communication when students require help from their peers or teachers. Very often, struggling students have great difficulty articulating where and why they are struggling. When all students and teachers use the same lexicon and guided pathways from the Calculations Template file, teachers are able to more quickly pinpoint problem areas and coach students through them.

\subsection{Clear expectations}

Students are encouraged prior to the laboratory session to review the $\mathbf{D}$ file in order to obtain a clear understanding of what data is expected to be gathered during the experiment; this prevents the situation where students leave the laboratory with an incomplete set of raw data, only to find that they cannot complete their calculations later. The A file clearly outlines the list of expected results, which helps prevent students from unintentionally submitting incomplete lab reports.

\section{BENEFITS FOR TEACHERS}

\subsection{Reduced grading effort}

One of the most significant advantages to using the TLDM system for teachers is the reduction in time spent grading reports. This reduction in grading effort and subsequent time savings can be redirected towards providing feedback on other aspects of lab reporting and returning graded reports to students more quickly. New teaching assistants can also use the TLDM system to familiarize themselves with the calculations associated with the experiment that they are assigned, so that they are ready to provide the best support for students.

\subsection{Ability to provide better feedback}

One of the major challenges with laboratory course education is that teachers cannot easily have the correct answers on hand, as they are specific to each student's unique set of raw data. Each time a particular student seeks help from a teacher, or while the teacher is grading a particular lab report, a lot of time is spent by the teacher doing repetitive calculations with a similar yet different set of raw data. Using the $\mathbf{C}$ file, teachers have instant access to the unique correct results for each student's experiment. Teachers can be more effective with these correct answers on hand to guide students through difficulties. The time saved can be used to have more value-added interactions with students. Teachers also have the option of pulling up the raw data, calculations and results anytime to support follow-up questions from students after they receive their graded reports.

The C file clearly delineates the correct path to calculating the correct final results. This allows teachers to visualize all major milestones, thereby helping them identify error points in student reports more quickly and efficiently. This also allows them to provide more focused feedback while grading by being able to easily include the correct calculations and answers found in the $\mathbf{C}$ file.

\subsection{Data collection for course improvement}

Since the D file must be submitted on the day of the experiment and is then used to generate the unique answer key for grading, any "fudging" of data or results can be readily identified. The system also enables teachers to provide an alternative raw data set (from other students or from past years) to any student who could not complete their experiments as planned, allowing these students to still work on the calculations and write their reports.

After using the TLDM system for some time, teachers will begin to build a repository of raw data and results from students. While some teachers have a habit of collecting similar information in the form photocopies of lab notebooks and reports, this information is difficult to 
access due to physical limitations (one must physically store, transport and flip through the pages) and inconsistencies in formatting (data tables between students are different in style and location). The TLDM system enables digital storage and access to the growing library of student data over the years, and most importantly, ensures that all data is formatted in the same style. This facilitates statistical analysis of this data for tracking student and equipment performance.

For example, armed with data from the past several years of running a particular experiment, a teacher can track the decreasing yield from a particular unit operation and deduce that there is something going wrong with the piece of equipment, which can initiate repairs to begin on the unit before a catastrophic failure occurs. In another example, the teacher can calculate the percentage of students obtaining the correct value for a set of results and find that one of the calculations may be particular challenging, which can prompt additional time and guidance to be provided there. All of this information can be used to troubleshoot issues, drive continual course improvement, as well as assist in periodic accreditation activities for the program.

\section{EXPANDED LEARNING OPPORTUNITIES}

Students are introduced to the growing trend of electronic data collection and processing. The traditional use of pen and paper for recording raw data and performing calculations is still used in some situations, but has largely been replaced with systems such as electronic laboratory notebooks (ELNs) and laboratory information management systems (LIMs) in industry. The main drivers for this transformation are reduced cost, improved data traceability, increased information accuracy and better accessibility to data, all of which are benefits to implementing electronic systems for education as well. Students who experienced the use of the TLDM system are better prepared for using these data systems when they begin work in industry.

\subsection{Effective use of spreadsheets}

Microsoft Excel ${ }^{\mathrm{TM}}$ and similar spreadsheet programs are routinely used in business. These programs can do far more than simply record data in a table, and can also perform complex mathematical operations. The Calculations Template file contains pre-formatted tables that help demonstrate effective use of Microsoft Excel ${ }^{\mathrm{TM}}$ for numerical operations. Students are able to learn by example strategies for spreadsheet layout, labeling and cell referencing, and they are encouraged and guided to explore and learn various Excel ${ }^{\mathrm{TM}}$ features to complete their calculations. Scaffold fading can be applied to prompts for proper spreadsheet use in the Calculations Template file, so that as students begin to master the use of Microsoft
Excel $^{\mathrm{TM}}$, supports can decrease and eventually disappear in more senior years.

\subsection{Data capture and reuse for new assignments}

Spreadsheet programs, like Microsoft Excel ${ }^{\mathrm{TM}}$, feature a large set of statistical and data analysis tools. These tools are important to the developing engineer, but there are limited opportunities for students to learn to apply these tools during their engineering education.

The TLDM system helps capture the large amounts of lab data that would have otherwise been forgotten and lost soon after the completion of experiments. Additional teaching assignments can be constructed to use this data.

For example, raw data generated for an experiment from one or more previous years can be provided to students to perform statistical operations to analyze the distribution of results. In another example, the raw data in all the $\mathbf{D}$ files submitted by the entire class in the current year can be provided to each student, who can then be asked to compare their individual results to the class average; in the event that their results differ significantly from the class average, they can be asked to hypothesize scientific reasons for the deviations.

In a related application, students can also be provided with sample raw data that represent particular operating conditions of significance, such as ideal scenarios or those where errors have occurred. In this example, the sample raw data can serve two purposes: 1) to assist in selfdirected learning along with the provided answers to sample raw data, and 2) to support additional assignment questions where students compare their own results against the answers to sample raw data and come up with scientific explanations to the differences.

The TLDM system provides a means for collecting and organizing big data from the lab, and also provides new avenues for students to learn scientific and mathematical concepts using this data.

\section{RESULTS}

The TLDM system was first introduced in September 2014 to the CHBE 262 Chemical Engineering and Applied Chemistry Laboratory course with 105 students. CHBE 262 includes experiments related to topics covered in the second-year level Chemical Engineering and Chemistry lecture courses. Students enrolled in this course come from a wide range of academic backgrounds and some might have had very limited laboratory experience, which made this course challenging to design and teach. Due to growing class sizes, teachers found it increasingly difficult to devote the necessary time to instruct individual students on how to carry out their calculations and data analysis. Prior to the introduction of the TLDM system, very lengthy and detailed step-by-step text-based documents were available on the course website, but these documents have been 
insufficient for some students. Additionally, many students have had very little prior experience with Microsoft Excel $^{\mathrm{TM}}$, which made some of the calculation tasks overwhelming. Some struggling students even resorted to changing their raw data in order to obtain desired results. It became impossible for teachers to manually check the accuracy of all reported raw data and results while grading reports. An immense amount of time was spent verifying calculations instead of discussing the conceptual underpinnings of the experiments with students. It is impossible to have deeper scientific conversations when determining the numerical accuracy of the results already takes most of the teacher's attention. The TLDM system changed that by efficiently determining the numerical accuracy of a student's results so that teachers can spend more time interacting with students on higher-level issues of understanding. The TLDM system files were made available to students through the campus Learning Management System.

In November 2014, approximately 3 months after the pilot introduction of the TLDM system, the Chemical and Biological Engineering (CHBE) Undergraduate Society conducted a voluntary online survey soliciting feedback on the TLDM System via social media for students enrolled in CHBE 262. This voluntary survey was completed by $24 \%$ of the 105 students enrolled in the course. The following statement is an excerpt from a letter issued by the CHBE Undergraduate Society summarizing the survey results:

"At the time of writing this letter, 25 students have replied and the majority of the feedback has been positive. The ability to have instant feedback on their calculations is the number one reason why students like the Excel ${ }^{\mathrm{TM}}$ templates. Overall, $96 \%$ of the students surveyed are in support of having the TLDM system in third and fourth year labs."

Based on the survey results and personal feedback from their classmates, they also concluded that:

"The TLDM System has greatly benefited the students in CHBE 262, with its ease of use and clarity. On behalf of the second year CHBE students, we advocate and support the development of the TLDM system for upper year labs and future students."

The statements from the CHBE Undergraduate Society show that the student feedback on the TLDM system is overwhelmingly positive. Students were also asked to provide feedback on specific features of the TLDM system and comment on their effectiveness. Figure 2 shows student feedback on the use of the TLDM system as well as approval of specific features:

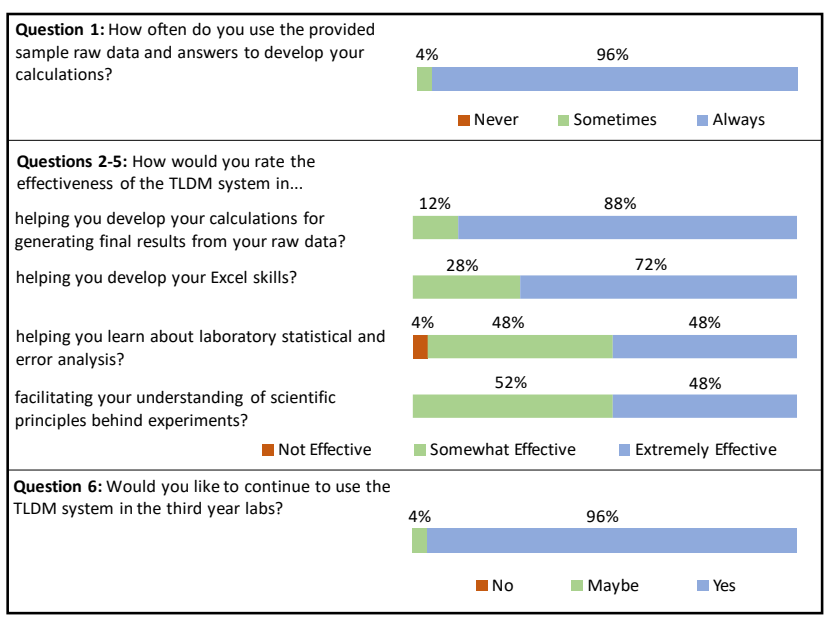

Figure 2: 2014 CHBE 262 student feedback on the effectiveness of various features of the TLDM system

An overwhelming majority (96\%) of students surveyed reported that they always used the provided sample raw data for self-directed learning, and none reported that they never used the system. Similarly, an overwhelming majority of students also recommended the continued use of the TLDM system. Almost all of the students reported that the system was either extremely effective or somewhat effective in helping them execute laboratory calculations, and learn how to use Microsoft Excel ${ }^{\mathrm{TM}}$, statistics and scientific principles. As expected, the greatest number of students (88\%) answered "extremely effective" regarding help with laboratory calculations, which is the primary aim of the majority of the components of TLDM system. A slightly reduced number of students (72\%) answered "extremely effective" regarding help with learning Excel ${ }^{\mathrm{TM}}$ skills, which is also expected as this learning objective is expected to take place in a more passive manner. It should be noted that dedicated assignments and prompts related to statistics have not yet been introduced in the CHBE 262 experiments at the time of this pilot launch of the system, which could explain why the least number of students found the system to be extremely effective in providing help in these areas.

Feedback on the TLDM system was also received from teaching assistants serving in CHBE 262. The following is an excerpt from this feedback:

"The TLDM system has been an incredible tool for my colleagues and I, helping us to track progress of students, grade lab reports, etc. The implementation of an automated program in conjunction with the current TLDM system is an important next-step for students, teaching assistants and course instructors."

Overall, teaching assistants especially enjoyed the significant time savings from grading lab reports when they use the TLDM system to generate unique answer keys to grade all numerical results. As intended, they found that they had more time to provide feedback on lab reports and 
felt more confident when guiding students through difficult calculations when they are armed with the correct calculations and results on hand.

At the time of the pilot introduction of the TLDM system, the $\mathbf{D}, \mathbf{A}$ and $\mathbf{C}$ files still had to be manually moved and opened by the user; this was a mundane operation that presented an opportunity for improvement through automation. Further development of the TLDM system has since occurred to include new components for the automatic manipulation system core files.

Following the success of its pilot introduction, the TLDM system continued to be implemented in CHBE 262 for subsequent years, and continued to receive positive feedback. In 2015, the TLDM system was introduced into CHBE third-year laboratory courses. The level of scaffolding offered by the TLDM system was reduced: the students were no longer provided with the Calculations Template file containing pre-made tables and instructions. Figure 3 below shows student feedback on the use of the TLDM system in the Term 1 of the CHBE 362 Process and Environmental Engineering Laboratory (Fall 2018) lab course with faded scaffolding:

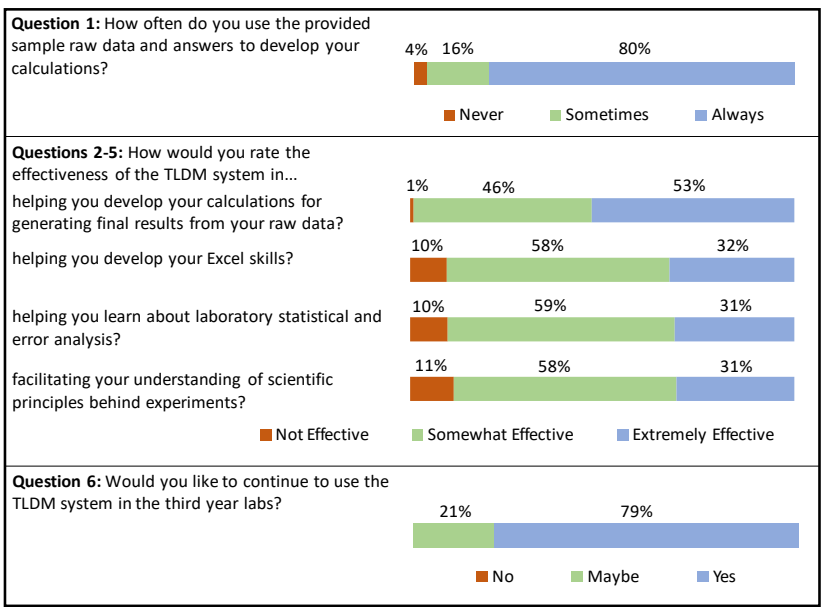

Figure 3: Term 1 CHBE 362 Process and Environmental Engineering Laboratory (Fall 2018) lab course student feedback on the effectiveness of various features of the TLDM system

Of the total of 136 students enrolled in the course, 114 students $(84 \%)$ participated in the survey. Overall approval of the TLDM system remained very high, with all of the students answering "yes" or "maybe" when asked whether they recommend the continued use of the system in the course. The majority (96\%) of students reported that they always or sometimes use the provided sample raw data and answers to develop their calculations, despite the fact that they are no longer provided with the Calculations Template file. It should be noted that all students enrolled in CHBE 362 should have already experienced the use of the TLDM system while they were enrolled in the prerequisite course, CHBE 262, where the system has already been implemented. Almost all of the students
(99\%) still reported that the TLDM system is effective in helping them through their calculations, but the percentage of students who answered "extremely effective" dropped from $88 \%$ in CHBE 262 (Figure 2) to only 53\% in CHBE 362. Similar trends were observed for questions about the effectiveness of the TLDM system in helping with the development of Excel ${ }^{\mathrm{TM}}$ skills, statistics and scientific concepts. These results are somewhat expected as fading has been applied to the scaffolding offered to students in CHBE 362, and it is the intent for the TLDM system to provide less support for the more advanced students in this course. These students have experienced the full level of scaffolding provided by the system in CHBE 262, so it is expected for them to recognize that it provided less help to them in CHBE 362. The overall positive feedback in CHBE 362 demonstrates how the TLDM system can be adapted to students operating at different levels.

\section{CONCLUSIONS}

The TLDM system helps students deal with the large amount of data generated in teaching laboratories by incorporating frequent feedback and flexible scaffolding to help them master complex numerical calculations. Teachers can use the TLDM system to improve the accuracy and efficiency of grading, thereby allowing additional time to be spent providing feedback to students. The traceability and accuracy of all laboratory data is improved, which increases the clarity and transparency of grading. Also, the collected data sets can be used to create new teaching opportunities, track student and equipment performance, and help with course development and program accreditation.

While the successful implementation of the TLDM system has been demonstrated in Chemical Engineering laboratory courses, it has strong potential for application to laboratory courses in other disciplines as well. As long as numerical data is generated in a teaching laboratory environment, the basic features of the TLDM system offered through its 5 core files can be applied to other disciplines and course settings.

Also, while the implementation of the TLDM system has been demonstrated based on Microsoft Excel ${ }^{\mathrm{TM}}$ as a calculations platform, it is potentially possible to implement most of its features on other spreadsheet applications. The concept of providing students with sample raw data and answers can also be applied to calculation tools other than spreadsheets, such as statistical or modeling software, but it is expected to be more difficult to implement the automatic grading component of the TLDM system in these applications.

The TLDM system allows you to add new rigour to your laboratory courses with reduced grading effort. The time savings allow the teacher to re-focus student/teacher conversations to the important underlying scientific principles behind each laboratory experiment. 


\section{Acknowledgments}

We gratefully acknowledge the financial support of The University of British Columbia Teaching and Learning Enhancement Fund (TLEF).

We would also like to extend our sincere gratitude to Dr. Peter Englezos, Head of Chemical and Biological Engineering for supporting the TLDM project, Dr. Louise Creagh for providing very valuable suggestions, the CHBE Undergraduate Society for their support and for conducting CHBE 262 survey, and Dr. Jonathan Verrett for allowing us to conduct the CHBE 362 survey.

\section{References}

[1] L. Feisel and A. Rosa, "The role of the laboratory in undergraduate engineering education," Journal of Engineering Education, vol. 94, no. 1, pp. 121-130, 2005.

[2] A. Hofstein and V. Lunetta, "The laboratory in science education: foundations for the twenty-first century," Science Education, vol. 88, no. 1, pp. 28-54, 2003.

[3] J. Ma and J. Nickerson, "Hands-on, simulated, and remote laboratories," ACM Computing Surveys, vol. 38, no. 3, pp. $1-24,2006$.

[4] E. Fereira, R. Lima and R. Salcedo, "Spreadsheets in chemical engineering education - a tool in process design and process integration," Int. J. Engng Ed., vol. 20, no. 6, pp. 928-938, 2004.

[5] S. A. Oke, "Spreadsheets applications in engineering education: a review," Int. J. Engng Ed., vol. 20, no. 6, pp. 893-901, 2004.

[6] M. Niazkar and S. H. Afzali, "Application of Excel spreadsheet in engineering education," Proc.of 1st International Conference on Engineering Education, 2015.

[7] R.M. Felder, "Teaching engineering in the $21 \mathrm{st}$ century with a 12th century teaching model: how bright is that?" Chem. Engr. Education, vol. 40, no. 2, pp. 110-113, 2006.

[8] S. Ambrose, M. Bridges, M. DiPietro, M. Lovett, and M. Norman, How Learning Works: Seven Research-Based Principles for Smart Teaching, San Francisco: John Wiley \& Sons, 2010

[9] K. A. Connor, Presentation, "Flipping a classroom: a continual process of refinement", Conf. American Society for Engineering Education, 2014 [Online]. Available: http://www.asee.org/file_server/papers/attachment/file/000 4/4535/ASEE_Flipped_Proces [Accessed: 03- Feb- 2019].

[10] Carl Wieman Science Education Initiative, "Motivating Learning".[Online document], Available:
http://www.cwsei.ubc.ca/resources/files/MotivatingLearning_CWSEI.pdf. [Accessed: 04- Feb- 2019].

[11] E.L. Bjork, and R.A. Bjork, "Making things hard on yourself, but in a good way: creating desirable difficulties to enhance learning," in M. A. Gernsbacher and J. Pomerantz, Eds., Psychology and the Real World: Essays Illustrating Fundamental Contributions to Society (2nd edition), New York: Worth, pp. 59-68, 2014.

[12] J.A. Ross, "Controlling variables: a meta-analysis of training studies," Review of Educational Research, vol. 58 no. 4, pp. 405-437, 1988.

[13] D. Wood, J.S. Bruner and G. Ross, "The role of tutoring in problem-solving," Journal of Child Psychology and Psychiatry, vol. 17, no. 2, pp. 89-100, 1976.

[14] D.R. Woods, A.N. Hrymak, R.R. Marshall, P.E. Wood, C.M. Crowe, T.W. Hoffman, J.D.Wright, P.A. Taylor, K.A. Woodhouse and C.G.K. Bouchard, "Developing problemsolving skills: the McMaster Problem Solving program," $J$. of Engineering Education, Vol. 86, no. 2, pp 75-91, 1997.

[15] C. Putnam, J. O'Donnell and N. Bertozzi, "Scaffolding and fading within and across a six-semester CDIO design sequence," Proceedings of the 6th International CDIO Conference, 2010.

[16] R. C. Clark, F. Nguyen, J. Sweller. Efficiency in Learning Evidence-Based Guidelines to Manage Cognitive Load, $\mathrm{p}$ 157. San Francisco: John Wiley \& Sons, 2006. 